\title{
Diagnostic Role of Dysregulated Circular RNA hsa_circ_0036722 in Laryngeal Squamous Cell Carcinoma
}

This article was published in the following Dove Press journal: OncoTargets and Therapy

\section{Yang Guo \\ Qiang Huang \\ Juan Zheng \\ Chi-Yao Hsueh \\ Xiaohui Yuan \\ Yu Heng \\ Liang Zhou}

Department of Otorhinolaryngology Head and Neck Surgery, Shanghai Key

Clinical Disciplines of

Otorhinolaryngology, Eye \& ENT

Hospital of Fudan University, Shanghai,

People's Republic of China
Correspondence: Liang Zhou

Department of Otorhinolaryngology

Head and Neck Surgery, Shanghai Key

Clinical Disciplines of

Otorhinolaryngology, Eye \& ENT Hospital

of Fudan University, No. 83, Fenyang

Road, Xuhui District, Shanghai 20003I,

People's Republic of China

Fax +86 2I-64377I34

Email zhoulent@I26.com
Purpose: Dysregulated circular RNAs (circRNAs) have been shown to play important roles in various cancers, and could serve as diagnostic biomarkers. However, research focusing on the roles of the circRNAs in laryngeal squamous cell carcinoma (LSCC) is limited. This research aimed to explore the expressions of hsa_circ_0036722 in LSCCs and its diagnostic significance.

Materials and Methods: The expression levels of the circular RNA, hsa_circ_0036722, and its parental gene, RHCG, in 41 pairs of LSCC tissues and paired adjacent normal tissues were validated with quantitative real-time polymerase chain reaction (qRT-PCR). The diagnostic values of hsa_circ_0036722 alone and combined with RHCG in LSCC were evaluated using receiver operating characteristic (ROC) curves. Bioinformatics analysis predicted likely cross-talk between hsa_circ_0036722 and RHCG. Then, qRT-PCR and luciferase reporter assay were used to examine the effect of hsa_circ_0036722 on miR-1248 and miR-1248 on RHCG expression. CCK-8 assays were conducted to investigate their effects on LSCC cell line.

Results: Hsa_circ_0036722 and RHCG were downregulated in LSCC tissues $(P<0.0001)$. The expression level of hsa_circ_0036722 was significantly correlated with the differentiation level of $\operatorname{LSCC}(P=0.018)$. The area under the ROC curve of hsa_circ_0036722 was 0.838 , which reached 0.859 when hsa_circ 0036722 was combined with RHCG as a biomarker. Mechanistically, hsa_circ_0036722 could directly sponge miR-1248 to antagonize its inhibitory effect on RHCG. And downregulation of hsa_circ_0036722 could promote the proliferation of LSCC cell line through upregulating miR-1248.

Conclusion: Our results indicated that hsa_circ_0036722 was downregulated in LSCC, which regulate the function of RHCG in LSCC via inhibiting miR-1248, and it could serve as a potential diagnostic marker for LSCC.

Keywords: circular RNA, diagnostic significance, laryngeal squamous cell carcinoma, biomarker

\section{Introduction}

As the sixth most prevalent cancer worldwide, head and neck cancer has become a growing burden for the public. ${ }^{1,2}$ Laryngeal cancer is the third most common head and neck cancer, and the vast majority is laryngeal squamous cell carcinoma (LSCC). ${ }^{3}$ Larynx carcinogenesis is influenced by both environmental and genetic factors. The most extensively established environmental risk factors for LSCC are smoking and excessive alcohol consumption. ${ }^{4}$ Various microRNAs (miRNAs) and long noncoding RNAs (lncRNAs) have been shown to be involved in the 
carcinogenesis and development of LSCC. ${ }^{2,5-8}$ Apart from miRNAs and lncRNAs, another type of non-coding RNA, called circular RNAs (circRNAs), has been demonstrated to play critical roles in LSCC. ${ }^{9}$

CircRNAs are covalently closed loops lacking $5^{\prime}$ caps and $3^{\prime}$ polyadenylation tails. ${ }^{10}$ Initially believed to be byproducts of splicing errors, circRNAs have now been shown to participate in different pathophysiological conditions, especially in various cancers. ${ }^{11-15}$ Generally, circRNAs function as "miRNA sponges" that bind to target miRNAs, suppressing their inhibiting effects on downstream mRNAs ${ }^{16}$ and forming various competing endogenous RNA (ceRNA) networks. In addition, some circRNAs can interact with RNA-binding proteins to regulate different physiological processes, such as apoptosis and the cell cycle. ${ }^{12,17}$ Some circRNAs can modulate the transcription, splicing and even translation of some genes. ${ }^{10,18}$ The most relevant functions of circRNAs for translational medicine could be their potential as biomarkers for diverse diseases. ${ }^{19}$

The distinctive closed loop structure, which is resistant to RNA exonucleases, makes circRNAs more stable than their cognate linear mRNAs. ${ }^{20}$ The characteristics of abundance and cell-, tissue-, developmental stage-, and diseasespecificity of circRNA makes them uniquely advantageous as biomarkers for facilitating the diagnosis and prognosis estimation of diseases. ${ }^{10,21}$ For example, hsa_circ_0001649 has been proposed as a potential biomarker for hepatocellular carcinoma ${ }^{22}$ and hsa_circ_0067934 may serve as biomarker for esophageal squamous cell carcinoma (ESCC). ${ }^{23}$ As far as potential circRNA biomarkers for head and neck cancer, most researches have focused on oral squamous cell carcinoma (OSCC); ${ }^{24-26}$ the diagnostic and prognostic roles of circRNAs in LSCC are still largely unknown.

In this study, we focused on hsa_circ_0036722, a circRNA that was suggested in microarray study to be dysregulated in LSCC. ${ }^{9}$ It is located at chr15:90,019,985-90,030,216 and its sample gene is Rh family $\mathrm{C}$ glycoprotein (RHCG), a confirmed tumor suppressor gene in various cancers. ${ }^{27,28}$ First, we validated downregulated expression of hsa circ_0036722 in LSCC tissues using quantitative real-time polymerase chain reaction (qRT-PCR). Second, we explored the relationships between hsa_circ_0036722 expression and clinicopathological characteristics in LSCC patients as well as its diagnostic value. Then, the potential LSCCassociated ceRNA network containing hsa_circ_0036722 and RHCG was predicted. Finally, the anti-tumor effect of hsa circ 0036722 through sponging miR-1248 to regulate RHCG in LSCC was validated.

\section{Materials and Methods}

\section{Patients and Specimens}

A total of 41 paired samples of LSCC and adjacent normal tissues were obtained from patients who underwent surgeries in Department of Otorhinolaryngology Head and Neck Surgery, Eye \& ENT Hospital of Fudan University between 2018 and 2019. No patients received radiotherapy or chemotherapy before surgery and all LSCC tissues were confirmed by pathologists. Clinical and pathological characteristics of patients were recorded. This study was approved by the Institutional Ethics Committee of the Eye \& ENT Hospital of Fudan University. Informed consent forms were signed by all patients before sample collection and the study was performed in accordance with the Declaration of Helsinki. All tissues were stored at $-80{ }^{\circ} \mathrm{C}$ until use.

\section{RNA Extraction and qRT-PCR Analyses}

TRIzol reagent (Invitrogen, Carlsbad, California, USA) was used to extract total RNA in tissues following manufacturer's instructions. Quantity and quality of RNA were examined with Nanodrop ND-2000 (Thermo Fisher Scientific, Inc). PrimeScript $^{\mathrm{TM}}$ RT Master Mix (TaKaRa, Dalian, China) was used to perform reverse transcription and SYBR Green Premix Ex Taq ${ }^{\mathrm{TM}}$ II (TaKaRa, Dalian, China) was used to perform qRT-PCR according to the manufacturer's instructions. GAPDH was chosen as the internal standard control, and values of $\Delta \mathrm{Ct}$ or $2^{-\Delta \Delta \mathrm{Ct}}$ were calculated to represent the relative expression of RNAs. Higher values of $\Delta \mathrm{Ct}$ or $2^{-}$ ${ }^{\Delta \Delta \mathrm{Ct}}$ represented relatively lower or higher expression levels, respectively. The primers used were as follows: forward primer of 5'-TCCAACTTTCTGTAGGGCTC-3' and reverse primer of 5'-GAAGCCTCCCAGTAGACC-3' for hsa_circ_0036722; forward primer of 5'-GGTTCC ACTTCTTACAAGACCG-3' and reverse primer of 5'GGGCTGACTTTACCCAGAACT-3' for RHCG; forward primer of 5'- CAATGACCCCTTCATTGACC-3' and reverse primer of 5'-TTGATTTTGGAGGGATCTCG-3' for GAPDH.

\section{Prediction of hsa_circ_0036722-miRNA- RHCG Network}

According to the "miRNA sponge" theory, hsa_circ_0036722 might regulate the expression of its parental gene, RHCG, by modulating the miRNAs targeting 
RHCG. To test this idea, we first predicted the potential miRNAs that could be bound by hsa_circ_0036722 using the Circular RNA Interactome (https://circinteractome.nia. nih.gov/) and RegRNA2.0 (http://regrna2.mbc.nctu.edu. tw/). The miRNAs targeting RHCG were forecasted with Targetscan (http://www.targetscan.org) and miRWalk 2.0 (http://mirwalk.umm.uni-heidelberg.de/). Finally, the miRNAs predicted to be involved in the hsa circ_0036722-miRNA-RHCG network were selected from the intersection of the above results.

\section{Cell Culture and Transfection}

AMC-HN-8 cells were cultured in RPMI-1640 (HyClone, Logan, UT) medium supplemented with 10\% FBS (Gibco, Grand Island, NY), 1\% streptomycin and penicillin, as well as $1 \%$ L-glutamine (Gibco, Grand Island, NY) at $37{ }^{\circ} \mathrm{C}$ in humidified air with $5 \% \mathrm{CO}_{2}$ as previously reported. ${ }^{7}$ And siRNA targeting hsa_circ_0036722 and miR-1248 inhibitor as well as negative controls (NCs) were obtained from Genomeditech (Shanghai, China). The transfection was completed with Lipofectamine 2000 (Invitrogen, Carlsbad, CA, USA) according to the manufacturer's instructions.

\section{Cell Counting Kit-8 (CCK-8) Assay}

CCK-8 assays were performed to determine cell proliferation abilities of various transfected cells. LSCC cell lines AMC-HN-8 transfected with indicated siRNAs, inhibitors or both were seeded at a concentration of $1 \times 10^{3}$ cells/well into 96-well plates and cultured in $37{ }^{\circ} \mathrm{C}$ under a $5 \% \mathrm{CO}_{2}$ atmosphere. Cell proliferation was measured by CCK-8 reagent (CCK8, Dojindo, Osaka, Japan) following the instructions of manufacturer.

\section{Luciferase Reporter Assay}

For hsa_circ_0036722-miR-1248 binding luciferase assays, the HEK-293T cells were co-transfected with miR-1248 mimics or NC combined with wild-type or mutated hsa_circ_0036722-luciferase reporter plasmids using Lipofectamine 2000; for the miR-1248-RHCG luciferase assay, the HEK-293T cells were co-transfected with miR1248 mimics or NC combined with wild-type or mutated RHCG-luciferase reporter plasmids (Genomeditech, Shanghai) using Lipofectamine 2000. Luciferase activity was detected using Dual-Glo Luciferase Assay system (Promega, Madison, WI) and then Firefly luciferase activities were normalized to the Renilla luciferase activities according to the manufacturers' protocols.

\section{Statistical Analysis}

GraphPad Prism 6.02 (GraphPad Software, La Jolla, CA, USA) and SPSS version 22.0 (SPSS Inc, Chicago, IL, USA) were used to perform the statistical analyses. The results of qRT-PCR were presented as mean \pm standard deviation (SD). Paired $t$-tests were used to compare the relative expression of hsa_circ_0036722 as well as RHCG in LSCC and adjacent normal tissues. The relationships between hsa_circ_0036722 expression levels and clinicopathological characteristics were assessed using $t$-test or one-way analysis of variance (ANOVA). The correlation between hsa_circ_0036722 and RHCG in LSCC patients was explored with Pearson correlation. The diagnostic values of hsa_circ_0036722 and RHCG were evaluated with ROC curves. $P$ value $<0.05$ was considered statistically significant.

\section{Results}

\section{Downregulated Expression of} hsa_circ_0036722 in LSCC Tissues

Hsa_circ_0036722 consists of exons 2-9 and its spliced sequence length is $1127 \mathrm{bp}$ (Figure 1), which is the longest among known exonic circRNAs generated from RHCG. ${ }^{29}$ Divergent primers were used to detect the expression levels of hsa_circ_0036722 in 41 pairs of LSCC tissues and adjacent normal tissues. The products of qRT-PCR were sequenced and the results of Sanger sequencing experiment validated the back-splice junction of hsa circ_0036722 (Figure 2A). In addition, hsa_circ_0036722 remain stable after RNase $\mathrm{R}$ treatment while its cognate linear mRNA RHCG were degraded (Figure 2B). And the results of the qRT-PCR assay indicated that the expression level of hsa circ 0036722 in LSCC was significantly lower than in paired adjacent mucosa tissues $(\mathrm{n}=41$, $P<0.0001$ ) (Figure 3A)

\section{Relationship Between hsa_circ_0036722 Expression Levels and Clinicopathological} Factors

Relationship between the clinicopathological factors of enrolled patients and the expression levels of hsa circ_0036722 was explored. Hsa_circ_0036722 expression levels were significantly associated with the pathological differentiation of LSCC $(P=0.018)$ (Table 1). However, no other clinicopathological factors, including age, $\mathrm{T}$ stage, $\mathrm{N}$ stage or tumor sizes, were associated with hsa_circ_0036722 expression. 


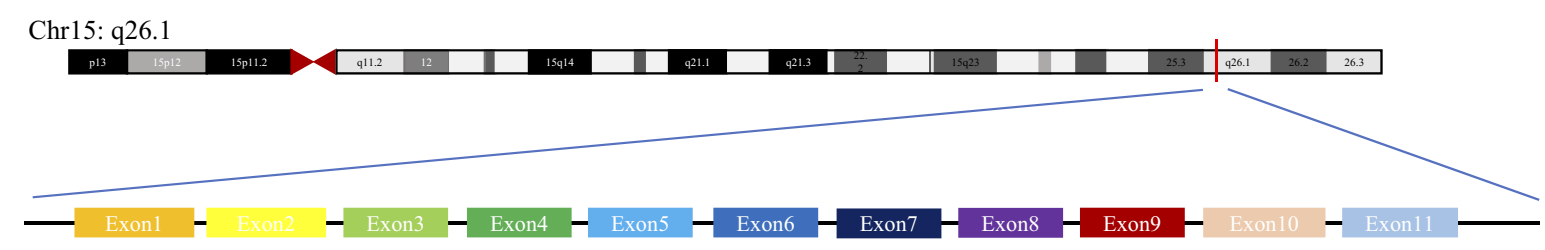

$\mathbf{a}$

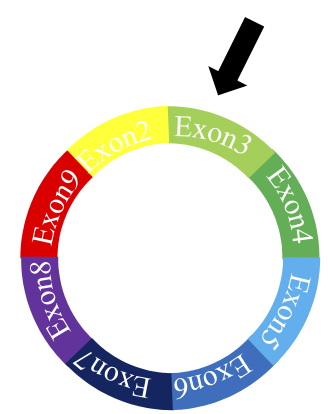

Hsa_circ_0036722 b

Figure I (A) Hsa_circ_0036722 is generated from chr I5:90,019,985-90,030,216 through back-splicing and is composed of eight exons spanning from exon 2 to 9 of RHCG. (B) Canonical splicing encodes the corresponding linear mRNA, RHCG.

A

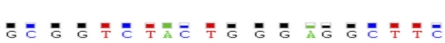

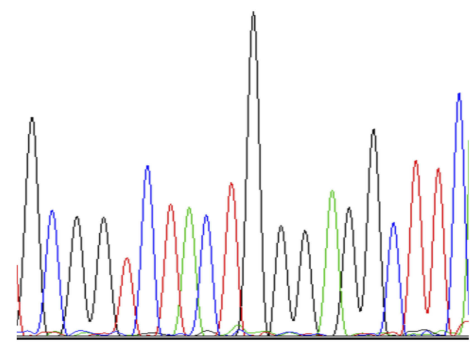

B

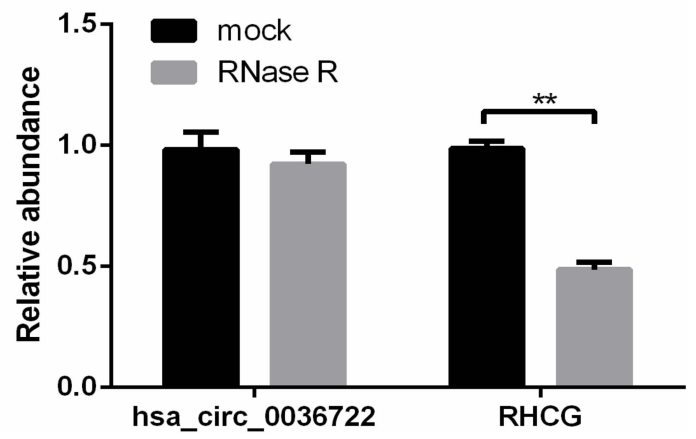

Figure 2 (A) The back-splicing junction of hsa_circ_0036722 was validated with Sanger sequencing experiments. (B) The stabilities of hsa_circ_0036722 and its cognate linear mRNA RHCG treated with RNase R was assessed by qRT-PCR (**P $<0.0 \mathrm{I})$.
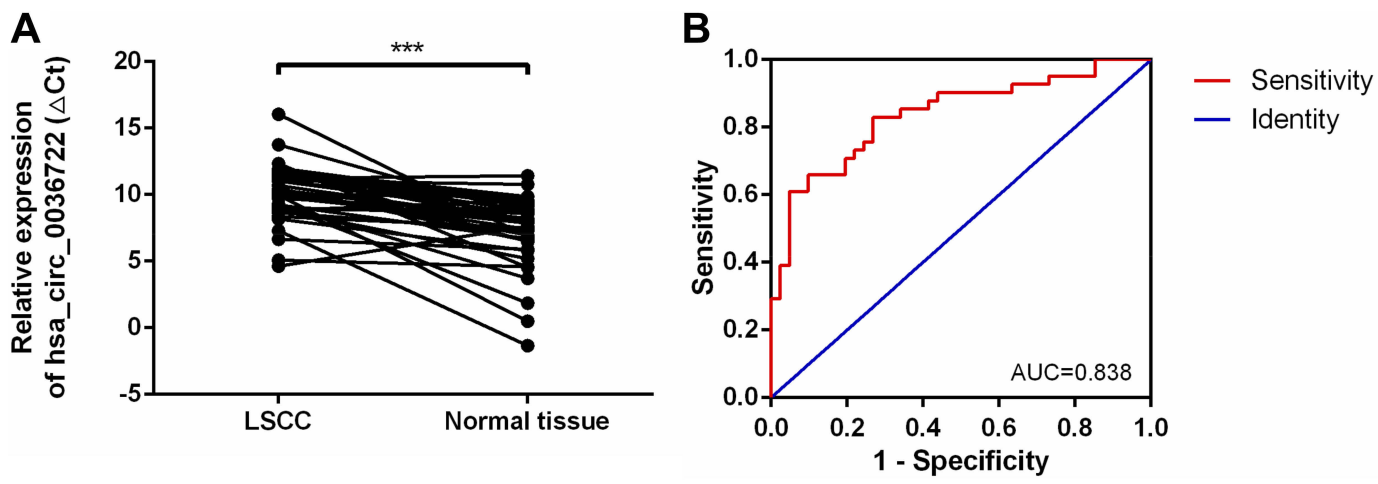

Figure 3 (A) Hsa_circ_0036722 was downregulated in LSCC tissues compared to paired adjacent normal tissues $(\mathrm{n}=4 \mathrm{I}$, $* * * P<0.000 \mathrm{I})$. Higher values of $\triangle$ Ct represent relatively lower expression levels. (B) The diagnostic potential of hsa_circ_0036722 in LSCC. The area under the ROC curve (AUC) was 0.838 (95\% Cl: 0.750 to $0.925, P<$ 0.0001 ). 
Table I Correlation Between Clinicopathological Factors and hsa_circ_0036722 Expression Levels $(\Delta C t)$ in Laryngeal Squamous Cell Carcinoma Patients

\begin{tabular}{|l|l|l|l|}
\hline Characteristics & $\begin{array}{l}\text { Number of } \\
\text { Patients }\end{array}$ & Mean \pm SD & P value \\
\hline $\begin{array}{l}\text { Age } \\
<60\end{array}$ & 13 & $9.77 \pm 1.91$ & 0.4482 \\
$\geq 60$ & 28 & $10.31 \pm 2.18$ & \\
\hline $\begin{array}{l}\text { Tumor sizes }(\mathrm{cm}) \\
<3\end{array}$ & 18 & $10.05 \pm 2.76$ & 0.8073 \\
$\geq 3$ & 23 & $10.21 \pm 1.43$ & \\
\hline $\begin{array}{l}\text { Differentiation } \\
\text { Well }\end{array}$ & 11 & $8.83 \pm 1.92$ & $0.0180 *$ \\
Moderate & 26 & $10.43 \pm 1.75$ & \\
Poor & 4 & $11.89 \pm 3.06$ & \\
\hline $\begin{array}{l}\text { T stage } \\
\text { TI-2 } \\
\text { T3-4 }\end{array}$ & 18 & $9.80 \pm 2.35$ & 0.2607 \\
\hline N stage & 23 & $10.41 \pm 1.88$ & \\
N0 & 25 & & \\
NI-3 & 16 & $10.44 \pm 2.54$ & \\
\hline
\end{tabular}

Note: *Indicated statistical significance.

\section{Diagnostic Value of hsa_circ_0036722 in LSCC}

Considering the significant relationship between the expression levels of hsa_circ_0036722 and the clinicopathological characteristics of LSCC patients, we performed receiver operating characteristic (ROC) curve analysis to investigate the diagnostic value of hsa_circ_0036722 expression. The area under the ROC curve (AUC) for hsa_circ_0036722 distinguishing LSCC from adjacent normal tissues was 0.838 (95\% confidence interval (CI): 0.750 to $0.925 ; P<0.0001$; Figure $3 \mathrm{~B}$ ), indicating its potential as a biomarker for LSCC.

\section{Downregulated Expression of RHCG and Its Diagnostic Value in LSCC Tissues}

Usually, the possible functions of circRNAs are associated with their parental genes. ${ }^{30-32}$ Thus, we hypothesized that the functions of RHCG could partly explain the probable functions of hsa_circ_0036722 in LSCC. The expression levels of RHCG in LSCC and paired adjacent normal tissues were explored by qRT-PCR. The results suggested that RGCG expression was significantly lower in LSCC than in adjacent normal tissues $(\mathrm{n}=41, * * * P<0.0001)$ (Figure 4A). The ROC curve was built to explore the diagnostic value of RHCG; the AUC of RHCG for differentiating LSCC from paired normal tissues was 0.808 (95\% CI: 0.713 to $0.904, P<0.0001$ ) (Figure 4B).

\section{Relationship Between hsa_circ_0036722} and RHCG, and Diagnostic Value of the Combination of hsa_circ_0036722 and RHCG in LSCC

As both hsa_circ_0036722 and RHCG were downregulated in LSCC tissues and hsa_circ_0036722 was generated from sample gene of RHCG, it was of interest to see if there was a correlation between the expression levels of hsa_circ_0036722 and RHCG. Pearson correlation analysis indicated the expression levels of hsa_circ_0036722 and RHCG were positively correlated in LSCC tissues $(r=0.6220, P<0.001)$ (Figure 5A). To explore the potential value of the combination of hsa_circ_0036722 and RHCG as a diagnostic biomarker, ROC curve analysis was conducted. The AUC of the combination reached 0.859 (95\% CI: 0.775 to $0.943, P<0.0001$ ), suggesting that the combination of the two markers was a better diagnostic biomarker for LSCC (Figure 5B).

\section{Potential ceRNA Network Involved in hsa_circ_0036722-RHCG Interactions}

To explore the likely ceRNA regulatory network involving hsa_circ_0036722 and RHCG, the miRNAs that can bind to both hsa_circ_0036722 and RHCG were bioinformatically predicted. The Circular RNA Interactome indicated that 52 miRNAs could be targeted by hsa_circ_0036722, while RegRNA 2.0 found fewer candidate miRNAs. Targetscan and miRWalk 2.0 showed that RHCG could be targeted by 1642 and 2313 miRNAs, respectively. MiR-1248 was the only miRNA predicted by all four website tools (Figure 6A). The results were represented with Venny 2.0 (http:/bioin fogp.cnb.csic.es/tools/venny/index.html); sequence matching between the predicted circRNA-miRNA-mRNA interactions are shown in Figure 6B. Thus, the hsa circ_0036722 might play a role in LSCC through the miR1248/RHCG axis. We next investigated the clinical significance of miR-1248 in head and neck squamous cell carcinoma (HNSCC) using an integrated online bioinformatic tool (http://kmplot.com/analysis/) established by Lánczky et al. ${ }^{33}$ Higher levels of miR-1248 predicted poorer prognosis of HNSCC patients, indicating that miR-1248 acted as oncogene in HNSCC (Figure 6C). According to the ceRNA theory, this was consistent with the tumor 

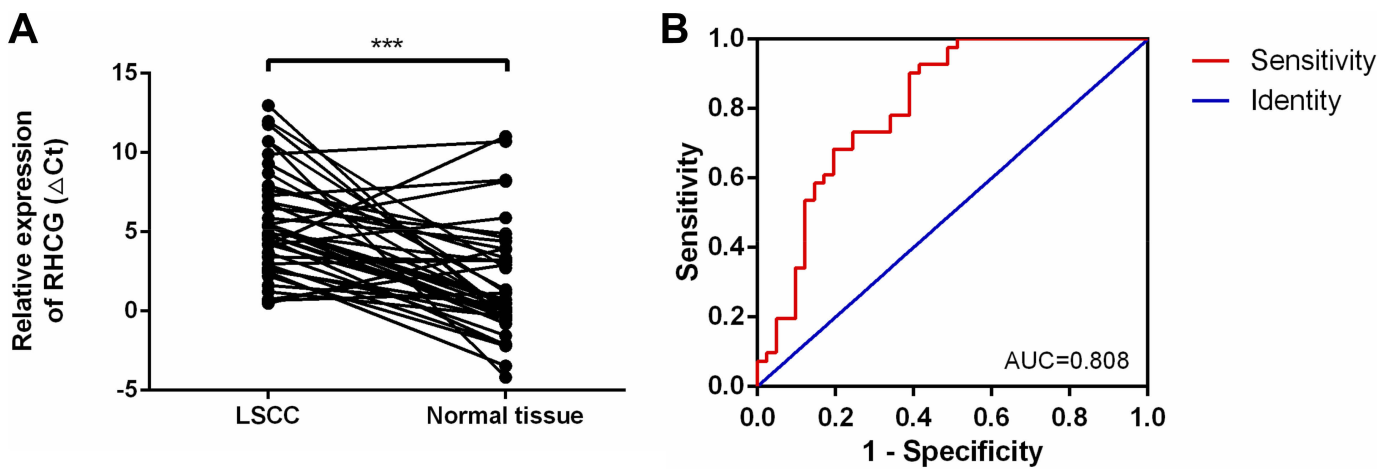

Figure 4 (A) RHCG was downregulated in LSCC tissues compared to paired adjacent normal tissues $(n=4 I, * * * P<0.000 I)$. Higher values of $\Delta C t$ represented relatively lower expression levels. (B) The diagnostic potential of RHCG in LSCC. The area under the ROC curve (AUC) was 0.808 ( $95 \%$ Cl: 0.713 to $0.904, P<0.0001$ ).
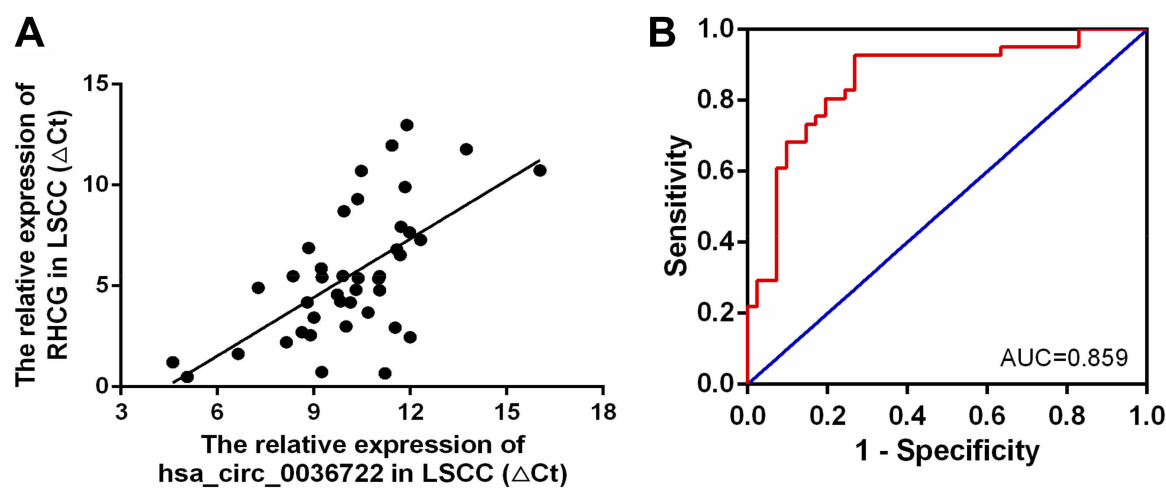

- Sensitivity

- Identity

Figure 5 (A) Expression levels of RHCG were significantly correlated with the expression levels of hsa_circ_0036722 in LSCC tissues $(r=0.6220, P<0.00 \mathrm{I})$. (B) The diagnostic potential of the combination of hsa_circ_0036722 and RHCG in LSCC. The area under the ROC curve (AUC) was 0.859 (95\% Cl: 0.775 to $0.943, P<0.000$ I).

suppressor roles of hsa_circ_0036722 and RHCG in LSCC considering their downregulation observed in this study.

\section{Hsa_circ_0036722 Functions Through miR-I 248/RHCG Axis in AMC-HN-8 LSCC Cells}

After transfection of siRNA targeting hsa_circ_0036722, the expression levels of hsa_circ_0036722 were downregulated as proved by qRT-PCR (Figure 7A). And the expression levels of miR-1248 and RHCG in AMC-HN-8 transfected with siRNAs targeting hsa_circ_0036722 were studied. The qRT-PCR results indicated that miR-1248 was upregulated while RHCG was downregulated after hsa_circ_0036722 was inhibited in AMC-HN-8 cells (Figure $7 \mathrm{~B}$ and $\mathrm{C}$ ). To investigate the role of miR-1248 in LSCC, AMC-HN-8 was transfected with miR-1248 inhibitors, and the transfection efficiency was examined by qRT-PCR (Figure 7D). In accordance with our prediction, the downregulation of miR-1248 significantly increased the expression of RHCG (Figure 7E). And co- transfection of miR-1248 inhibitors could partly contradict with RHCG decrease induced by downregulated hsa_circ_0036722 (Figure 7F). Furthermore, luciferase reporter assays were performed to confirm the relationship between miR-1248 and hsa_circ_0036722/RHCG, respectively. The data illustrated that miR-1248 upregulation significantly restrained the activity of hsa_circ_0036722WT reporter and RHCG-WT reporter (Figure $7 \mathrm{G}$ and H).

\section{Ablation of hsa_circ_0036722 Promote the Proliferation of AMC-HN-8 LSCC Cells Which Could Be Partly Reversed by the Inhibition of miR-I248}

CCK-8 assay showed that the proliferation ability of AMC-HN-8 transfected with siRNAs targeting hsa_circ_0036722 was enhanced compared to controls (Figure 8A). The proliferation ability of AMC-HN-8 transfected with miR-1248 inhibitors was significantly decreased compared to negative controls (Figure 8B). And it worth noting that co-transfection of miR-1248 
A

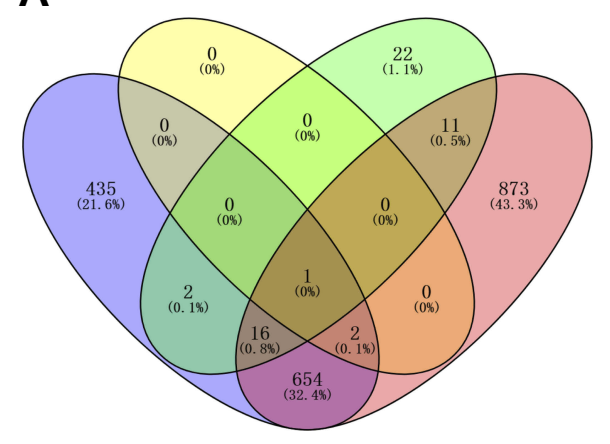

B

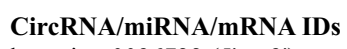

hsa_circ_0036722 (5' ... 3')

hsa-miR-1248 (3' ... 5')

3' UTR of RHCG (5' ... 3')

hsa_circ_0036722 (5' ... 3')

hsa-miR-1248 (3' ... 5')

3' UTR of RHCG (5' ... 3')
Predicted sequence pairing UCCAGUGCCCUGCACAAGAAGGG

|| || | | ||| |

AAAUCGUGUCACGAAUAUG-UUCUUCCA

|| | ||||||

CACUGUAAAUGCUAG-------AAGAAGGA

UUGAAGUCUAUGGAAAAGAAGGG

| | | | | |

AAAUCGUGUCACGAAUAUG-UUCUUCCA

|||||||||| $\mid$
Site type

7 mer-m8

8 mer

$7 \mathrm{mer}-\mathrm{m} 8$

8 mer

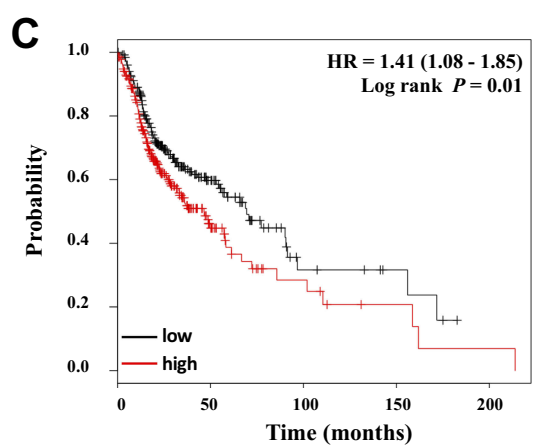

Figure 6 (A) MiR-1248 was predicted to be able to bind to both hsa_circ_0036722 and RHCG. Green area and yellow area indicate predictions from Circular RNA Interactome and RegRNA 2.0, respectively, of which miRNAs could be potentially sponged by hsa_circ_0036722; blue area and red area represent the potential miRNAs targeting RHCG predicted by Targetscan and miRWalk 2.0, respectively. (B) Predicted sequences pairing in the hsa_circ_0036722/miR-I248/RHCG ceRNA network. Hsa_circ_0036722 contains two predicted binding sites for miR-1248 and RHCG had one predicted 8-mer type binding site for miR-1248. (C) Higher levels of miR-I248 predict poorer prognosis of head and neck squamous cell carcinoma (HNSCC) patients.

inhibitors in AMC-HN-8 transfected with siRNA targeting hsa_circ_0036722 could partly reverse enhanced proliferation ability induced by hsa_circ_0036722 downregulation (Figure 8C).

\section{Discussion}

Despite tremendous research efforts and huge therapeutic advancements in LSCC, overall survival of this cancer has not been improved in the past years. ${ }^{34,35}$ Considering the poor prognosis and negative impact on the quality of life of LSCC patients, researches into potential biomarkers might shed a light on the early detection and improvement of therapy for LSCC.

Although they were discovered about 40 years ago, the important roles played by circRNAs have only recently been appreciated. ${ }^{36,37}$ Various circRNAs have now been validated as involved in different cancers. ${ }^{14,38-40}$ Considering the stability and the spatio-temporal specificity of circRNAs, they are optimal biomarkers for cancer diagnostics. ${ }^{14,19,41}$ The downregulated hsa_circ_0008309 and hsa_circ_001242 have been shown to be novel biomarkers and therapy targets in OSCC. ${ }^{25,26}$ Similarly, decreased expression of hsa_circ_0000190 and hsa_circ_002059 could serve as biomarkers for gastric cancer. $^{42,43}$ However, although a few studies have examined the dysregulation of circRNAs in LSCC, ${ }^{9,10}$ research about circRNA biomarkers in LSCC remains rare.

Here, we first validated the downregulation of hsa circ_0036722 in LSCC tissues compared with paired normal tissues. Examining the relationships between clinicopathological factors and the hsa_circ_0036722 expression levels suggested that as the differentiation levels became poorer, the expression levels of hsa_circ_0036722 also decreased. This suggested that hsa circ_0036722 expression levels were negatively associated with LSCC differentiation. ROC curve analysis illustrated that hsa_circ_0036722 could serve as a diagnostic biomarker for LSCC with AUC of 0.838 .

Many circRNAs were found to participate in cancer progression by affecting their parental genes. ${ }^{30,32,44-46} \mathrm{We}$ therefore wondered if the parental gene of hsa circ_0036722 was dysregulated in LSCC. The qRT-PCR indicated RHCG was downregulated in LSCC. This was consistent with the role of RHCG as tumor suppressor 
A

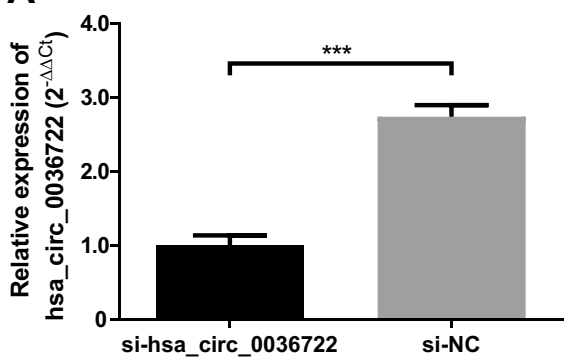

C

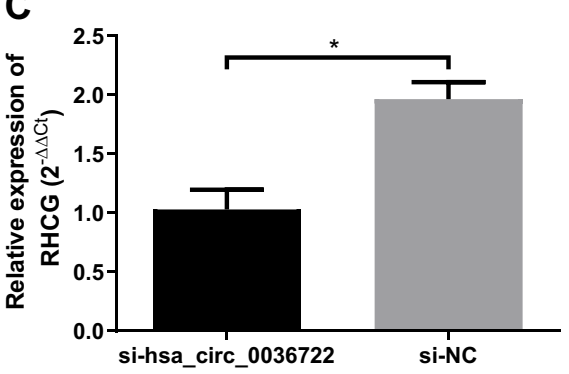

E

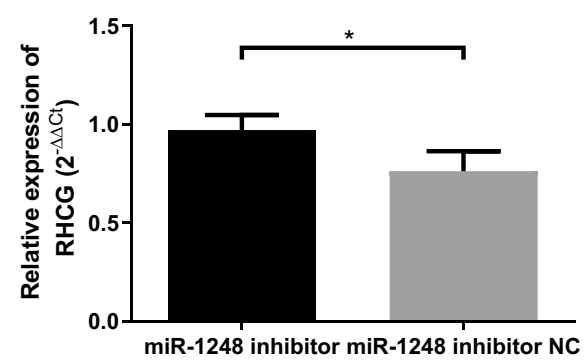

G
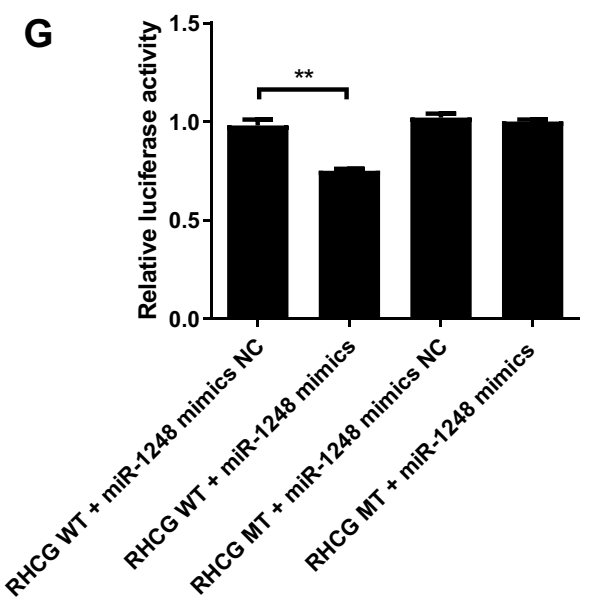

B

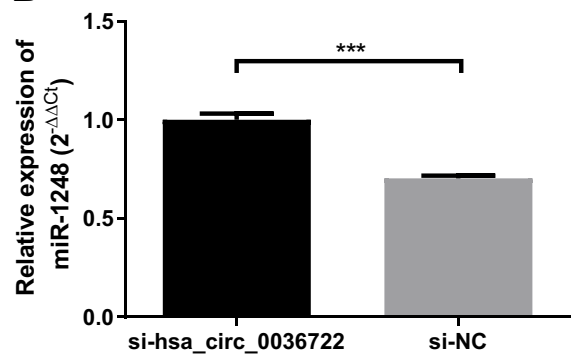

D

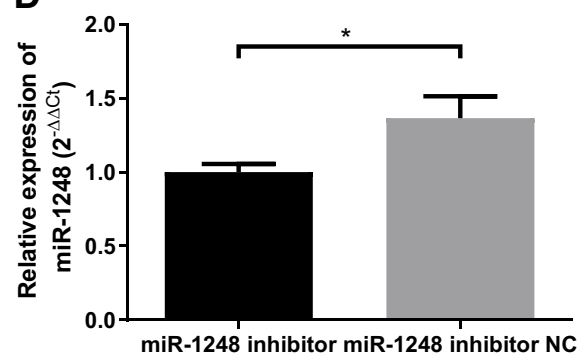

F

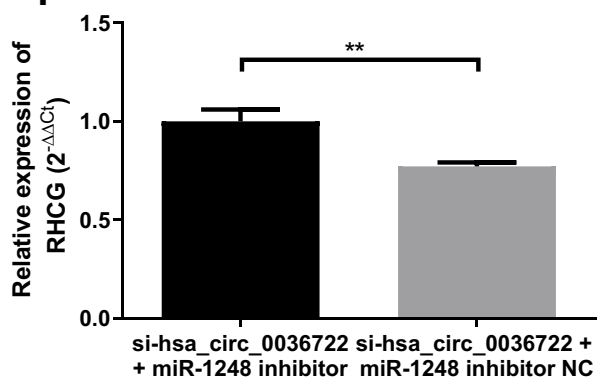

H

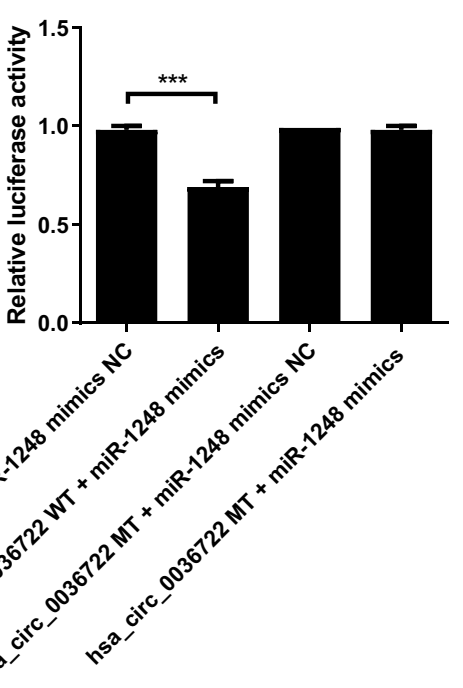

Figure 7 (A) Hsa_circ_0036722 was downregulated in AMC-HN-8 cell line transfected with siRNA targeting its junction sequence (****P < $0.000 \mathrm{I})$. (B and C) MiR-I248 was upregulated $(* * * P<0.0001)$ while RHCG $(* P<0.05)$ was downregulated after hsa_circ_0036722 was downregulated in AMC-HN-8 cell line. (D) The expression of miR-1248 was decreased by miR- 1248 inhibitors, and the transfection efficiency was examined by $q R T-P C R(* P<0.05)$. (E) Downregulation of miR- 1248 significantly increased the expression of RHCG $(* P<0.05)$. (F) Co-transfection of miR-1248 inhibitors could partly reverse the RHCG decrease induced by downregulated hsa_circ_0036722 (**P < $0.0 \mathrm{I})$. Higher values of $2^{-\Delta \Delta C t}$ represented relatively higher expression levels in this figure. $(\mathbf{G}$ and $\mathbf{H})$ Luciferase reporter assay showed that ectopic expression of miR-I248 could suppress the activity of hsa_circ_0036722-WT $(* * * P<0.001)$ and RHCG-WT $(* * P<0.01)$ reporters, respectively. 

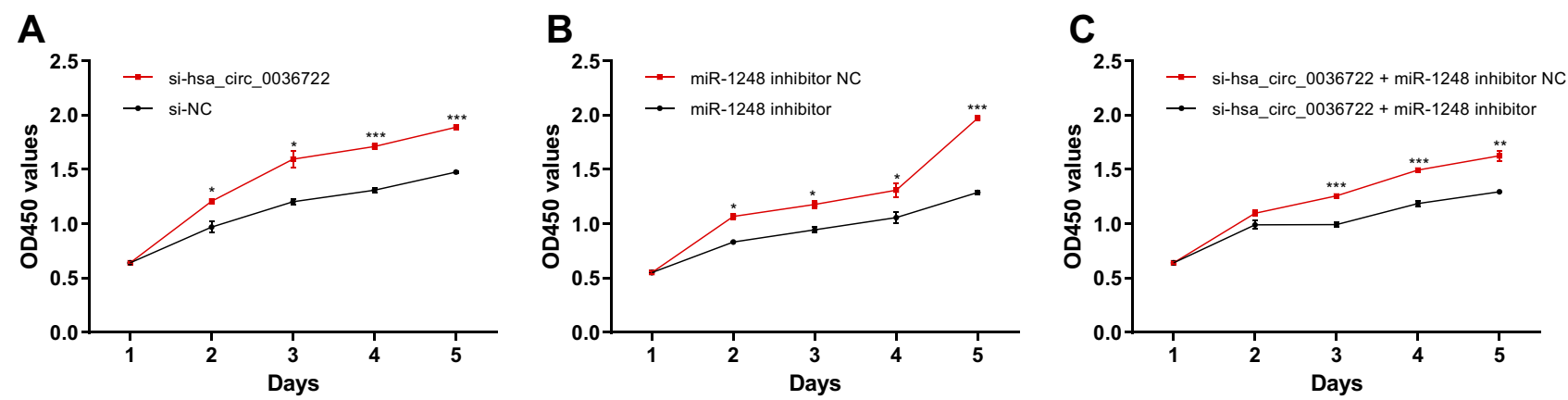

Figure 8 (A) CCK-8 assay showed that the proliferation ability of AMC-HN-8 transfected with siRNAs targeting hsa_circ_0036722 was enhanced compared to controls $(* P<0.05 ; * * * P<0.00 \mathrm{I})$. (B) The proliferation ability of AMC-HN-8 was inhibited by miR-1 248 inhibitors compared to negative controls $(* P<0.05 ; * * * P<0.001)$. (C) Inhibition of miR-1248 rescued the enhanced proliferation ability induced by hsa_circ_0036722 downregulation $(* * P<0.01$; ***P $<0.00 \mathrm{I})$.

gene in another subtype of HNSCCs, tongue squamous cell carcinoma. ${ }^{47}$ Similarly, the expression of RHCG was decreased in ESCC and correlated with poorer differentiation, invasion, lymph node metastasis and poorer prognosis, and RHCG could inhibit the tumorigenicity and progression of ESCC in-vitro and in-vivo via suppressing MMP1 expression. ${ }^{27}$ In addition, RHCG was found to be downregulated in cervical cancer and could induce apoptosis and suppress migration ability of cervical cancer cells by lowering TGF- $\beta 1$ expression. ${ }^{28}$

It is worth noting that the decreased expression of RHCG in LSCC was positively correlated with the expression of hsa_circ_0036722. The ROC curve analysis suggested that RHCG could distinguish LSCC from adjacent normal tissues with an AUC value of 0.808. Though this value was lower than that of hsa_circ_0036722 (AUC = 0.838), the addition of RHCG to hsa_circ_0036722 improved the diagnostic value, with the AUC of the combination reaching 0.859 . Thus, hsa_circ_0036722 could serve as diagnostic biomarker for LSCC, and could do so more efficiently if combined with RHCG.

In diverse cancers, circRNAs act as transcriptional regulators, protein decoys and templates for translation. ${ }^{48}$ In addition, most circRNAs function as "miRNA sponges" by targeting downstream miRNAs, which in turn affecting their target mRNAs. ${ }^{38}$ In most cases, the cognate linear mRNAs from the parental genes that produce the circRNAs are regulated at the end of circRNA/miRNA/mRNA axes..$^{30,44,46,49-51}$ A typical example is the anti-oncogenic circ-ITCH. In lung cancer, circ-ITCH sponges miR-7 and miR-214 to increase the expression of its parental gene, ITCH, which suppresses the progression of lung cancer via inhibiting the Wnt $/ \beta$ catenin pathway. ${ }^{30}$ This regulation mechanism also applies to colorectal cancer. ${ }^{44}$ Considering the ceRNA network theory and the positive correlation between hsa_circ_0036722 expression and RHCG expression, we hypothesized that hsa_circ_0036722 might partly influence the course of LSCC via regulating its anti-oncogenic parental gene RHCG through shared miRNAs.

One miRNA, miR-1248, that could bind to both hsa_circ_0036722 and RHCG was predicted by all four bioinformatics databases. A recent study suggested that miR-1248 played an oncogenic role in osteosarcoma by inhibiting apoptotic protein angiotensin II type 1 receptor (AGTR1) at the post-transcriptional level and was correlated with poorer survival and chemotherapy resistance. ${ }^{52}$ Furthermore, higher expression of miR-1248 was associated with BRCA1/2 mutation-associated hereditary breast cancer. ${ }^{53}$ To investigate the role of miR-1248 in LSCC, the relationship of miR-1248 expression with the prognosis of HNSCC patients was explored based on a web-tool that could analyze data from 523 HNSCC patients. ${ }^{33}$ The result indicated that the prognosis of HNSCC patients with higher expression of miR-1248 was poorer. Thus, miR-1248 was also an oncogene in HNSCC. This was in line with the predicted tumor suppressor roles of the decreased hsa_circ_0036722 and RHCG in LSCC.

Furthermore, we conducted CCK-8 assays that demonstrated the tumor-suppressor roles of hsa_circ_0036722 and oncogenic roles of miR-1248 in LSCC cell line. Downregulation of hsa_circ_0036722 could increase the expression of miR-1248, while RHCG could be upregulated by miR-1248 inhibition. What's more, luciferase reporter assays illustrated the direct interactions between miR-1248 and hsa_circ_0036722 as well as RHCG. Thus, we concluded 
that hsa_circ_0036722 sponges miR-1248, inhibiting the suppression effect of miR-1248 on its parental gene, RHCG, to function as tumor suppressor in LSCC.

\section{Conclusion}

Our study revealed that hsa_circ_0036722 was downregulated in LSCC compared with adjacent normal tissues and its expression levels were significantly associated with the differentiation levels of LSCC. For the first time, we demonstrated the diagnostic value of hsa_circ_0036722 for LSCC. In addition, its expression was associated with the expression of its parental gene, RHCG. The diagnostic value of the combination of RHCG and hsa_circ_0036722 was higher than that of hsa_circ_0036722 alone. Further function experiments indicated that hsa_circ_0036722 inhibit LSCC proliferation through sponging miR-1248 to regulate RHCG. Our results suggested that downregulated hsa_circ_0036722 could serve as a potential diagnostic biomarker for LSCC.

\section{Acknowledgment}

The present study was supported by grants from the Science and Technology Commission of Shanghai Municipality (19411961300 and 16411950100), Shanghai Shen Kang Hospital Development Center (SHDC12015114).

\section{Disclosure}

The authors report no conflicts of interest in this work.

\section{References}

1. Marur S, Forastiere AA. Head and neck squamous cell carcinoma: update on epidemiology, diagnosis, and treatment. Mayo Clin proc. 2016;91(3):386-396. doi:10.1016/j.mayocp.2015.12.017

2. Ahmad P, Sana J, Slavik M, Slampa P, Smilek P, Slaby O. MicroRNAs involvement in radioresistance of head and neck cancer. Dis Markers. 2017;2017:8245345. doi:10.1155/2017/8245345

3. Surono A, Priyanto P, Indrasari SR. Hypoxia-inducible Factor-1 $\alpha$ expression in Indonesian laryngeal squamous cell carcinoma patients. J Oncol. 2016;2016:3215463. doi:10.1155/2016/3215463

4. van Dijk BA, Karim-Kos HE, Coebergh JW, Marres HA, de Vries E. Progress against laryngeal cancer in The Netherlands between 1989 and 2010. Int J Cancer. 2014;134(3):674-681.

5. Cao P, Zhou L, Zhang J, et al. Comprehensive expression profiling of microRNAs in laryngeal squamous cell carcinoma. Head Neck. 2013;35 (5):720-728.

6. Zhang XW, Liu N, Chen S, et al. High microRNA-23a expression in laryngeal squamous cell carcinoma is associated with poor patient prognosis. Diagn Pathol. 2015;10:22.

7. Zheng J, Xiao X, Wu C, et al. The role of long non-coding RNA HOTAIR in the progression and development of laryngeal squamous cell carcinoma interacting with EZH2. Acta Otolaryngol. 2017;137(1):90-98.
8. Huang Q, Yang J, Zheng J, Hsueh C, Guo Y, Zhou L. Characterization of selective exosomal microRNA expression profile derived from laryngeal squamous cell carcinoma detected by next generation sequencing. Oncol Rep. 2018.

9. Zhao R, Li FQ, Tian LL, et al. Comprehensive analysis of the whole coding and non-coding RNA transcriptome expression profiles and construction of the circRNA-IncRNA co-regulated ceRNA network in laryngeal squamous cell carcinoma. Funct Integr Genomics. 2019;19(1):109-121.

10. Guo Y, Yang J, Huang Q, et al. Circular RNAs and their roles in head and neck cancers. Mol Cancer. 2019;18(1):44. doi:10.1186/s12943019-1003-5

11. Fan L, Cao Q, Liu J, Zhang J, Li B. Circular RNA profiling and its potential for esophageal squamous cell cancer diagnosis and prognosis. Mol Cancer. 2019;18(1):16. doi:10.1186/s12943-018-0936-4

12. Du WW, Fang L, Yang W, et al. Induction of tumor apoptosis through a circular RNA enhancing Foxo3 activity. Cell Death Differ. 2017;24 (2):357-370. doi:10.1038/cdd.2016.133

13. Fu L, Jiang Z, Li T, Hu Y, Guo J. Circular RNAs in hepatocellular carcinoma: functions and implications. Cancer Med. 2018;7 (7):3101-3109. doi:10.1002/cam4.1574

14. Luo Y H, Zhu X Z, Huang K W, et al. Emerging roles of circular RNA hsa_circ_0000064 in the proliferation and metastasis of lung cancer. Biomed Pharmacother. 2017;96:892-898. doi:10.1016/j. biopha.2017.12.015

15. Tan S, Sun D, Pu W, et al. Circular RNA F-circEA-2a derived from EML4-ALK fusion gene promotes cell migration and invasion in non-small cell lung cancer. Mol Cancer. 2018;17(1):1. doi:10.1186/ s12943-018-0887-9

16. Wang L, Tong X, Zhou Z, et al. Circular RNA hsa_circ_0008305 (circPTK2) inhibits TGF- $\beta$-induced epithelial-mesenchymal transition and metastasis by controlling TIF $1 \gamma$ in non-small cell lung cancer. Mol Cancer. 2018;17(1):1. doi:10.1186/s12943-018-0889-7

17. Du WW, Yang W, Liu E, Yang Z, Dhaliwal P, Yang BB. Foxo3 circular RNA retards cell cycle progression via forming ternary complexes with p21 and CDK2. Nucleic Acids Res. 2016;44 (6):2846-2858. doi:10.1093/nar/gkw027

18. Wilusz JE. A $360^{\circ}$ view of circular RNAs: from biogenesis to functions. Wiley Interdis Rev. 2018;9(4):e1478. doi:10.1002/wrna.1478

19. Meng S, Zhou H, Feng Z, et al. CircRNA: functions and properties of a novel potential biomarker for cancer. Mol Cancer. 2017;16(1):94. doi:10.1186/s12943-017-0663-2

20. Zheng Q, Bao C, Guo W, et al. Circular RNA profiling reveals an abundant circHIPK3 that regulates cell growth by sponging multiple miRNAs. Nat Commun. 2016;7(1):11215. doi:10.1038/ncomms11215

21. Yao J, Hu -L-L, Li X-M, et al. Comprehensive circular RNA profiling of proliferative vitreoretinopathy and its clinical significance. Biomed Pharmacother. 2019;111:548-554. doi:10.1016/j.biopha.2018.12.044

22. Li W H, Song Y C, Zhang H, et al. Decreased expression of hsa_circ_00001649 in gastric cancer and its clinical significance. Dis Markers. 2017;2017:4587698. doi:10.1155/2017/4587698

23. Xia W, Qiu M, Chen R, et al. Circular RNA has_circ_0067934 is upregulated in esophageal squamous cell carcinoma and promoted proliferation. Sci Rep. 2016;6(1):35576. doi:10.1038/srep35576

24. Su W, Wang Y, Wang F, et al. Circular RNA hsa_circ_0007059 indicates prognosis and influences malignant behavior via AKT/ mTOR in oral squamous cell carcinoma. J Cell Physiol. 2019;234 (9):15156-15166. doi:10.1002/jcp.28156

25. Li B, Wang F, Li X, Sun S, Shen Y, Yang H. hsa_circ_0008309 may be a potential biomarker for oral squamous cell carcinoma. Dis Markers. 2018;2018:7496890. doi:10.1155/2018/7496890

26. Sun S, Li B, Wang Y, et al. Clinical significance of the decreased expression of hsa_circ_001242 in oral squamous cell carcinoma. Dis Markers. 2018;2018:6514795. doi:10.1155/2018/6514795 
27. Ming X-Y, Zhang X, Cao -T-T, et al. RHCG suppresses tumorigenicity and metastasis in esophageal squamous cell carcinoma via inhibiting NF- $\kappa$ B signaling and MMP1 expression. Theranostics. 2018;8 (1):185-198. doi:10.7150/thno.21383

28. Wang D G, Li T M, Liu X. RHCG suppresses cervical cancer progression through inhibiting migration and inducing apoptosis regulated by TGF- $\beta 1$. Biochem Biophys Res Commun. 2018;503 (1):86-93. doi:10.1016/j.bbrc.2018.05.183

29. Glazar P, Papavasileiou P, Rajewsky N. CircBase: a database for circular RNAs. Rna. 2014;20(11):1666-1670. doi:10.1261/rna.043687.113

30. Wan L, Zhang L, Fan K, Cheng Z X, Sun Q C, Wang J J. Circular RNA-ITCH suppresses lung cancer proliferation via inhibiting the Wnt/ $\beta$ Catenin pathway. Biomed Res Int. 2016;2016:1579490. doi:10.1155/2016/1579490

31. Li X, Wang J, Zhang C, et al. Circular RNA circITGA7 inhibits colorectal cancer growth and metastasis by modulating the Ras pathway and upregulating transcription of its host gene ITGA7. J Pathol. 2018;246(2):166-179. doi:10.1002/path.5125

32. Hirsch S, Blatte TJ, Grasedieck S, et al. Circular RNAs of the nucleophosmin (NPM1) gene in acute myeloid leukemia. Haematologica. 2017;102(12):2039-2047. doi:10.3324/haematol.2017.172866

33. Lánczky A, Á Á, Bottai G, et al. miRpower: a web-tool to validate survival-associated miRNAs utilizing expression data from 2178 breast cancer patients. Breast Cancer Res Treatment. 2016;160 (3):439-446. doi:10.1007/s10549-016-4013-7

34. Steuer CE, El-Deiry M, Parks JR, Higgins KA, Saba NF. An update on larynx cancer. CA Cancer J Clin. 2017;67(1):31-50. doi:10.3322/ caac. 21386

35. Matulka M, Konopka A, Mroczko B, et al. Expression and concentration of matrix metalloproteinase 9 and tissue inhibitor of matrix metalloproteinases 1 in laryngeal squamous cell carcinoma. Dis Markers. 2019;2019:9. doi:10.1155/2019/3136792

36. Hansen TB, Jensen TI, Clausen BH, et al. Natural RNA circles function as efficient microRNA sponges. Nature. 2013;495 (7441):384-388. doi:10.1038/nature11993

37. Galasso M, Costantino G, Pasquali L, et al. Profiling of the predicted circular RNAs in ductal in situ and invasive breast cancer: a pilot study. Int J Genomics. 2016;2016:4503840.

38. Zhong Y, Du Y, Yang X, et al. Circular RNAs function as ceRNAs to regulate and control human cancer progression. Mol Cancer. 2018;17 (1):79.

39. Wang Y, Mo Y, Gong Z, et al. Circular RNAs in human cancer. Mol Cancer. 2017;16(1):25.
40. Zhang X, Zhou H, Jing W, et al. The circular RNA hsa circ 0001445 regulates the proliferation and migration of hepatocellular carcinoma and may serve as a diagnostic biomarker. Dis Markers. 2018;2018:3073467.

41. Wang F, Wang J, Cao X, Xu L, Chen L. hsa_circ_0014717 is downregulated in colorectal cancer and inhibits tumor growth by promoting p16 expression. Biomed Pharmacother. 2018;98:775-782.

42. Li P, Chen S, Chen H, et al. Using circular RNA as a novel type of biomarker in the screening of gastric cancer. Clin Chim Acta. 2015;444:132-136.

43. Chen S, Li T, Zhao Q, Xiao B, Guo J. Using circular RNA hsa_circ_0000190 as a new biomarker in the diagnosis of gastric cancer. Clinica Chimica Acta. 2017;466:167-171.

44. Zhou Y, Huang G, Zhu H, et al. Cir-ITCH plays an inhibitory role in colorectal cancer by regulating the Wnt/ $\beta$-Catenin pathway. PLoS One. 2015;10(6):e0131225.

45. Zhang HD, Jiang LH, Hou JC, et al. Circular RNA hsa_circ_0052112 promotes cell migration and invasion by acting as sponge for miR-125a-5p in breast cancer. Biomed Pharmacother. 2018;107: $1342-1353$.

46. Hou JC, Xu Z, Zhong SL, et al. Circular RNA circASS1 is downregulated in breast cancer cells MDA-MB-231 and suppressed invasion and migration. Epigenomics. 2019;11(2):199-213.

47. Ye H, Yu T, Temam S, et al. Transcriptomic dissection of tongue squamous cell carcinoma. BMC Genomics. 2008;9:1.

48. Shang Q, Yang Z, Jia R, Ge S. The novel roles of circRNAs in human cancer. Mol Cancer. 2019;18(1):6.

49. He R, Liu P, Xie X, et al. CircGFRA1 and GFRA1 act as ceRNAs in triple negative breast cancer by regulating miR-34a. J Exp Clin Cancer Res. 2017;36(1):145.

50. Bai N, Peng E, Qiu X, et al. CircFBLIM1 act as a ceRNA to promote hepatocellular cancer progression by sponging miR-346. J Exp Clin Cancer Res. 2018;37(1):172.

51. Lv T, Miao YF, Jin K, et al. Dysregulated circular RNAs in medulloblastoma regulate proliferation and growth of tumor cells via host genes. Cancer Med. 2018;7(12):6147-6157.

52. Zhao Y, Xu K, Liu P. Post-transcriptional control of angiotensin II type 1 receptor regulates osteosarcoma cell death. Cell Physiol Biochem. 2018;45(4):1581-1589.

53. Tanic M, Yanowski K, Gomez-Lopez G, et al. MicroRNA expression signatures for the prediction of BRCA1/2 mutation-associated hereditary breast cancer in paraffin-embedded formalin-fixed breast tumors. Int J Cancer. 2015;136(3):593-602.
OncoTargets and Therapy

\section{Publish your work in this journal}

OncoTargets and Therapy is an international, peer-reviewed, open access journal focusing on the pathological basis of all cancers, potential targets for therapy and treatment protocols employed to improve the management of cancer patients. The journal also focuses on the impact of management programs and new therapeutic agents and protocols on patient perspectives such as quality of life, adherence and satisfaction. The manuscript management system is completely online and includes a very quick and fair peer-review system, which is all easy to use. Visit http://www.dovepress.com/ testimonials.php to read real quotes from published authors. 